\title{
Potential Electrochemical Coronary Artery Disease Diagnosis Based on A Periostin Immunoassay
}

\author{
Xiu-Feng Zheng ${ }^{1}$, Yuan-Yuan Wang $^{2}$, Lei Han ${ }^{3}$, Xi-Chun Wang ${ }^{4}$, Shi-Lei Zhao ${ }^{5}$ and Hui Zou ${ }^{1 *}$ \\ ${ }^{1}$ Department of Cardiology, Heilongjiang Hospital, Harbin, Heilongjiang, P.R. China. \\ ${ }^{2}$ Department of Cardiology. Mudanjiang Medical University Hongqi Hospital, Mudanjiang, \\ Heilongjiang, P.R. China. \\ ${ }^{3}$ Department of Respiratory. Mudanjiang Medical University Hongqi Hospital, Mudanjiang, \\ Heilongjiang, P.R. China. \\ ${ }^{4}$ Department of Gerontological Neurology, Heilongjiang Hospital, Harbin, Heilongjiang, P.R. China. \\ ${ }^{5}$ Department of Nephrology, The First Affiliated Hospital of Harbin Medical University, Harbin, \\ Heilongjiang, P.R.China \\ *E-mail: $\underline{87888829 @ q q . c o m}$
}

doi: $10.20964 / 2017.01 .65$

Received: 25 October 2016 / Accepted: 29 November 2016 / Published: 12 December 2016

The preparation of a sensitive amperometric immunosensor for periostin was achieved. The synthesis of golg nanoprobe was carried out through depositing the gold nanoparticles in situ on silica nanophere functionalized by polydopamine, which was followed to label the signal antibodies. The method of covalent immobilization, in which the antibodies were captured on the glassy carbon electrode, was employed for the preparation of the immunosensor. The cyclic voltammetry (CV) was employed to investigate the performance of the decorated electrode at different modification stages. Furthermore, the study of the performance of the immunosensor was carried out in detail. The prepared immunosensor demonstrates highly sensitive for periostin detection with excellent correlation of detection ranging from 0.1 to $150.0 \mathrm{ng} / \mathrm{mL}$, where limit of $0.06 \mathrm{ng} / \mathrm{mL}$ can be detected, and estimated through a signal-to-noise ratio of 3 . The periostin be detected by one-step immunoassay in the proposed method, which acts as valuable role to diagnose the clinical early coronary artery disease.

Keywords: Coronary heart disease; Immunosensor; Periostin; Gold nanoparticles; Polydopamine

\section{$\underline{\text { FULL TEXT }}$}

(C) 2017 The Authors. Published by ESG (www.electrochemsci.org). This article is an open access article distributed under the terms and conditions of the Creative Commons Attribution license (http://creativecommons.org/licenses/by/4.0/). 